\title{
Comprehensive Evaluation of Geological and Engineering Sweet Spots of Shale Gas Reservoir: A Case Study of the Luzhou Block, Sichuan Basin
}

\author{
Yuan Wang ${ }^{*}$, Jianfa $\mathrm{Wu}^{2}$, Li Fu${ }^{1}$, Haoyong Huang², Gang Chen ${ }^{1}$, Cheng Chang \\ ${ }^{1}$ CNPC Engineering Technology R \& D Company Limited, Beijing, China \\ ${ }^{2}$ Southwest Oil and Gas Field Company of CNPC, Chengdu, China \\ Email: *wangyuandr@cnpc.com.cn
}

How to cite this paper: Wang, Y., Wu, J.F., Fu, L., Huang, H.Y., Chen, G. and Chang, C. (2021) Comprehensive Evaluation of Geological and Engineering Sweet Spots of Shale Gas Reservoir: A Case Study of the Luzhou Block, Sichuan Basin. International Journal of Geosciences, 12, 1021-1039.

https://doi.org/10.4236/ijg.2021.1211054

Received: November 5, 2021

Accepted: November 13, 2021

Published: November 16, 2021

Copyright $\odot 2021$ by author(s) and Scientific Research Publishing Inc. This work is licensed under the Creative Commons Attribution International License (CC BY 4.0).

http://creativecommons.org/licenses/by/4.0/

\begin{abstract}
There is a huge amount of marine shale gas resources in the southern Sichuan Basin in China, and most of the resources are at the buried depth of 3500 4500 meters. At present, deep shale gas is in the early stage of exploration and development. In order to achieve large-scale efficient development, in addition to optimizing favorable blocks, it is also to identify the optimal target in the vertical direction combine geology, drilling, and fracturing. Therefore, Taking the Longmaxi formation shale in the Luzhou block as the research object, based on drilling, logging, and core experiment data, through single well and 3D geomechanical modeling methods, analyze the characteristics of organic matter abundance, porosity, pore pressure, collapse pressure, mineral composition and in-situ stress of different layers of shale in Longmaxi formation. Systematically summarized the main controlling factors of the "sweet spot" of deep shale gas and establish the comprehensive evaluation system of deep shale gas "sweet spots", to clarify the optimal "sweet spots" of geology, drilling, and fracturing in the Longmaxi reservoir. Results show that the total organic carbon content, porosity, and gas saturation of the long111 layer are higher than other layers. The Long111 layer has a low collapse pressure and a high compressive strength, the risk of wellbore instability is relatively low. The stress difference coefficient of All layers is less than 0.3, and the brittleness index of the Long111 layer is $62.35 \%$. A complex fracture network is easier to form after fracturing. The conclusion shows that the Long111 layer is the optimal reservoir section of the Longmaxi Formation. Ensure the drilled rate of the Long111 layer and maximize the length of the horizontal section can obtain higher production.
\end{abstract}




\section{Keywords}

Deep Shale Gas, Geological Sweet Spots, Engineering Sweet Spots, Geomechanical Modeling

\section{Introduction}

As an important unconventional energy source, shale gas has become a hot spot in global resource development. In recent years, with the continuous development and improvement of basic theory and exploration and development technologies of shale gas in China, effectively developed have been made in shale gas resources at the buried depth of 2000 - 3500 meters [1] [2] [3] [4] [5]. With the continuous deepening of shale gas exploration and development in PetroChina, the potential of deep shale gas resources at the buried depth of $3500-4500$ have been gradually clarified. According to the evaluation results of shale gas resources, the favorable area of shale gas resources in the Sichuan Basin at the buried depth of 2000 - 4500 meters is about $18,000 \mathrm{~km}^{2}$ and the amount of shale gas resources are nearly 9.6 trillion $/ \mathrm{m}^{3}$, of which shale gas resources at the buried depth of 3500 - 4500 meters account for $86 \%$. Realizing the effective utilization of deep shale gas resources is an important foundation for realizing the mid-long term development plan of shale gas, and it will also make an important contribution to global energy security and economic development [6].

Shale gas is a typical continuous, large-area accumulation of natural gas, which is mainly distributed in high-quality shale layers in the center of the sedimentary basin and slope areas. It exceeds the concept of "reservoir" of conventional oil and gas and cannot find obvious trap boundaries. Therefore, exploration strategies need to change from finding "oil and gas reservoirs" to finding "sweet spot". The "sweet spots" of shale gas refer to the optimal area or optimal horizon for shale gas exploration and development, where is suitable for horizontal well drilling, scale fracturing, and realizing large-scale commercial exploitation. Horizontally, "sweet spot" refers to the enriched area of unconventional oil and gas with commercial exploitation value. Vertically, it refers to sections of organic-rich shale that can form industrial exploitation value after fracturing. There are many factors that affect shale gas "sweet spot". In the early stage of exploration, more attention was paid to the quality of static indicators. With the continuous deepening of exploration and development, the importance of engineering quality research has gradually been realized.

Based on previous research results, the author systematically summarized the main controlling factors of the "sweet spot" of deep shale gas, and clarified that the "sweet spot" of deep shale gas is mainly controlled by three factors [7] [8] [9] [10]: 1) Superior reservoir indexes (total organic carbon content, thermal maturity, total porosity, gas saturation, pore pressure, permeability, high-quality reservoir thickness, etc.), which are the material basis of shale gas enrichment. 2) 
Good drilling conditions (rock drillability, wellbore stability, etc.), which are the key to rapid production of shale gas. 3) Favorable fracturing quality (brittleness index, Young's modulus, Poisson's ratio, stress difference coefficient, natural fracture development degree, etc.), which are the core of efficient shale gas development.

The Luzhou block is the main development area for deep shale gas resources in China which is located in the southern Sichuan low fold belt, the scale of the faults in the area is relatively small. Its location is shown in Figure 1. The Longmaxi Formation reservoirs are continuously and stably distributed in the block, the buried depth is generally between $3500-4500 \mathrm{~m}$, and the deposition thickness is generally between $500-650 \mathrm{~m}$, which is much higher than the shale gas resources at the buried depth of 2000 - 3500 meters. The Longmaxi Formation is conform-able contact with the underlying Upper Ordovician Wufeng Formation and the Overlying Upper Lower Silurian Shiniulan Formation, and divided into the Long 2 member, the Long 12 sub-member, and the Long11 sub-member from top to bottom [11] [12]. However, compared with shale gas resources at the buried depth of 2000 - 3500 meters, deep shale gas has a more complicated tectonic background, higher pore pressure, and greater horizontal stress and stress difference. The drilling cycle of the $8-1 / 2$ in wellbore of the early drilled well is increased by $40 \%$ compared with the shale gas resources at the buried depth of 2000 - 3500 meters, and the single well production varies greatly. Therefore, it has important theoretical value and practical significance to evaluate the geology, drilling, and fracturing "sweet spots" of deep shale gas reservoirs.

Many previous studies have focused on the geological characteristics of reservoirs and the selection of favorable areas in the Luzhou block [13] [14] [15] [16] [17], however, the research on the selection of "sweet spots" combination of geology, drilling, and fracturing to identify the optimal target has gained less

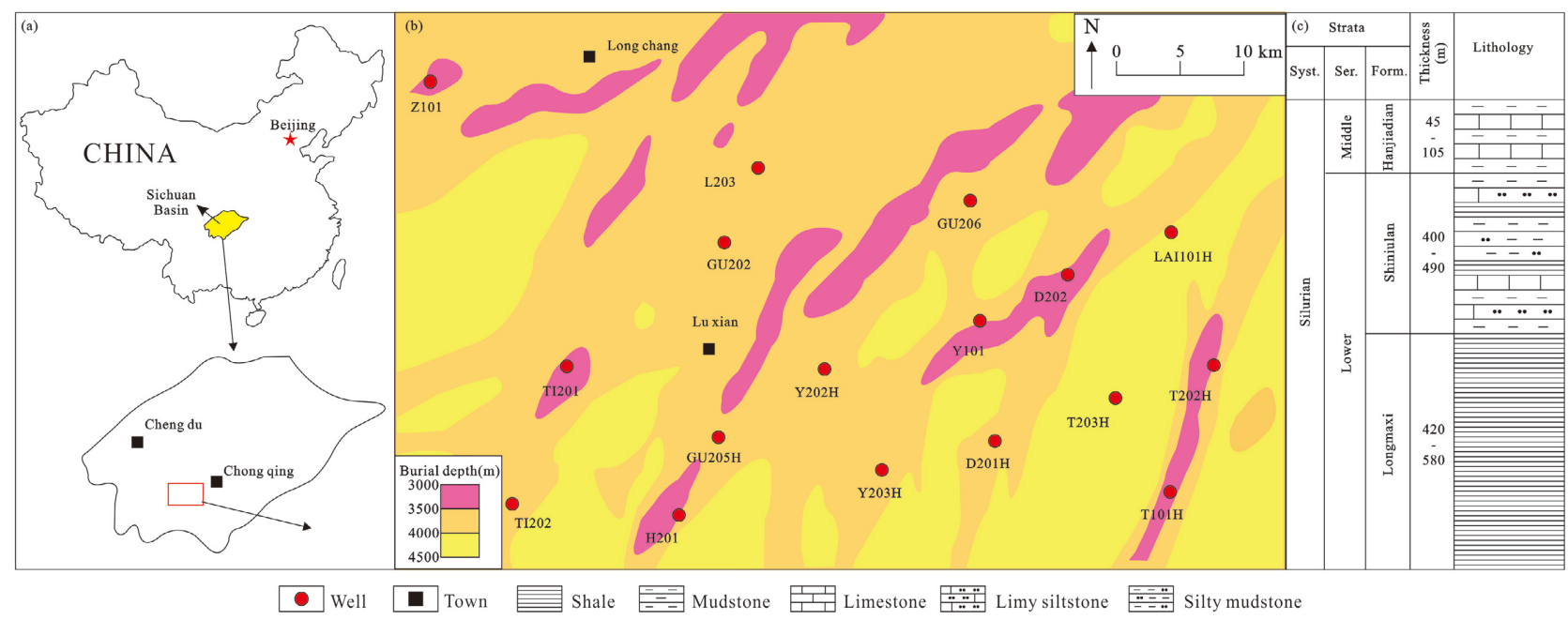

Figure 1. Geographic location and lithological column of study area. (a) Location of Sichuan basin; (b) Location of the study area; (c) Lithological column of the Silurian formation. 
attention. In this study, we take the Longmaxi formation shale in the Luzhou block as the research object, based on drilling, logging, and core experiment data, through single well and 3D geomechanical modeling methods, systematically study the geochemical characteristics, rock mechanics characteristics, brittleness characteristics, and in-situ stress characteristics of the shale gas reservoir in the block. Referring to national and industry standards of evaluation method of shale gas, combined with the specific condition of deep shale gas exploration and development practice, the comprehensive evaluation system of deep shale gas "sweet spots" is established around three main control factors (Table 1), to clarify the optimal target for the beneficial development of deep shale gas, and expected to provide a basis for the exploration and development of deep shale gas.

\section{Sample and Method}

\subsection{Sample Collection and Experimental Method}

A total of 106 fresh shale core samples from the Longmaxi formation were collected from eight wells for analysis, which were all carefully packed and directly delivered to the laboratories for the experiments.

TOC content, mineralogical composition, porosity, and rock mechanics parameters of all samples were measured in the laboratory. A LECOCS-230 was adopted for measuring TOC content of shales following China National Standards GB/T 19145-2003, and mineral content measurement was performed on a RIGAKU D/Max 2500PC X-ray diffractometer according to the SY/T5163-2018 standard. A Pore Master GT60 Mercury injection apparatus was used for mercury intrusion porosimetry experiment to measure the porosity of shales. The uniaxial compression test and triaxial compression test of shales were utilized to obtain the elasticity and strength parameters, such as Young's modulus, Poisson's ratio, and uniaxial compressive strength.

Table 1. Comprehensive evaluation parameter table of "sweet" spot.

\begin{tabular}{cccccc}
\hline \multirow{2}{*}{$\begin{array}{c}\text { Sweet } \\
\text { spots type }\end{array}$} & $\begin{array}{c}\text { Main } \\
\text { controlling } \\
\text { factors }\end{array}$ & Parameter & \multicolumn{3}{c}{ Level } \\
\cline { 3 - 6 } Geological \\
sweet spot & $\begin{array}{c}\text { Reservoir } \\
\text { property }\end{array}$ & PoC, \% & $>3.0$ & $3.0-2.0$ & $<2.0$ \\
& & Gas saturation, \% & $\geq 60$ & $60-50$ & $50-40$ \\
\hline \multirow{2}{*}{$\begin{array}{c}\text { Engineering } \\
\text { sweet spot }\end{array}$} & $\begin{array}{c}\text { Drilling } \\
\text { conditions }\end{array}$ & Collapse pressure & $<1.7$ & $1.7-1.8$ & $>1.8$ \\
& Fracturing & Rock drillability & $1-3$ & $4-6$ & $7-8$ \\
& quality & Young's modulus, Gpa & $>30$ & $30-20$ & $<20$ \\
& & Poisson's ratio & $<0.2$ & $0.2-0.25$ & $>0.25$ \\
& & Brittleness index, \% & $>60$ & $60-40$ & $<40$ \\
& & Stress difference coefficient & $0-0.3$ & $0.3-0.5$ & $>0.5$ \\
\hline
\end{tabular}




\subsection{Modeling Method}

\subsubsection{Rock Mechanics Parameters}

According to the logging data and the calculation model of rock mechanics parameters, Young's modulus, Poisson's ratio, uniaxial compressive strength, and other rock mechanics parameters can be calculated. And the calculated rock mechanics parameters can be revised according to the results of rock mechanics experiments. The calculated rock mechanical parameter profiles are shown in Figure 2.

\subsubsection{Pore Pressure}

A pore pressure prediction method considering the overpressure mechanism is pro-posed by Bowers [18]. It is necessary to determine the formation mechanism of abnormal high pressure in the Longmaxi formation in Luzhou block. Use the normally compacted mudstone data to establish a normal trend line of the relationship between compressional velocity and density, this can be expressed by the following equation:

$$
V_{P}=3420+454\left(\rho_{r}-1.27\right)^{4.03}
$$

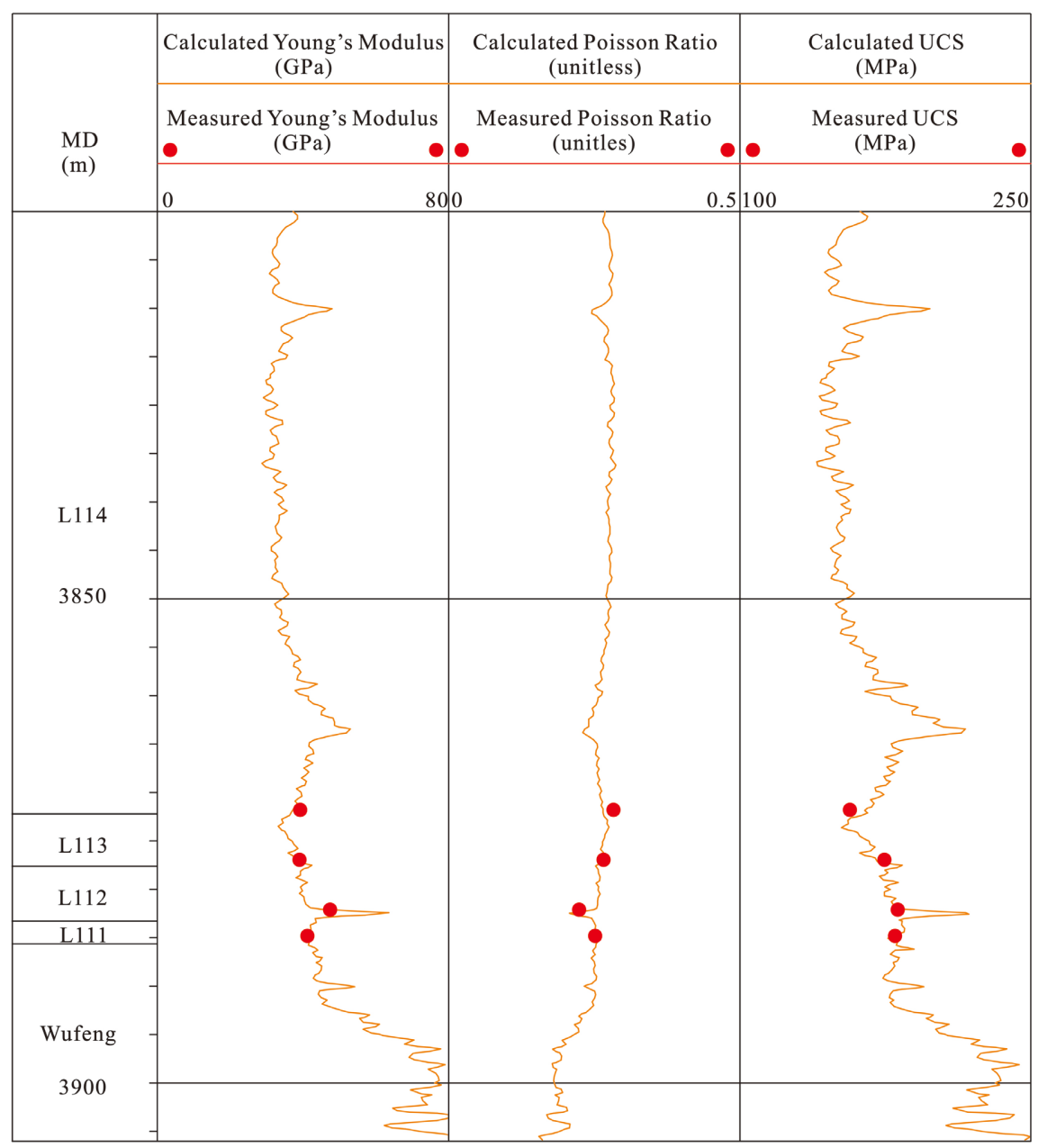

Figure 2. Rock mechanical parameter profiles. 
where $V_{P}$ is the compressional velocity, $\mathrm{m} / \mathrm{s} ; \rho_{r}$ is the density, $\mathrm{g} / \mathrm{cm}^{3}$.

The reduction degree of the compressional velocity of the Longmaxi formation in the Luzhou block is greater than the reduction degree of the density. The scatter points of compressional velocity-density deviate from the normal compaction trend line, and fall on the low-value direction of compressional velocity as shown in Figure 3. The abnormal high pressure of Longmaxi formation is due to pore fluid expansion. Therefore, this paper adopts the calculation formula of abnormal high pressure caused by the unloading mechanism proposed by Bowers, and calculate the model coefficients from logging data and core experiment data, which can be expressed by the following equation:

$$
P_{p}=P_{o}-\frac{\left(\sigma_{\max }^{\prime}\right)^{(1-U)}+\left(\frac{V_{p}-3420}{A}\right)^{(U / B)}}{Z}
$$

where $P_{p}$ is the pore pressure, $\mathrm{MPa} ; P_{o}$ is the overburden pressure, $\mathrm{MPa}$; $\sigma_{\max }^{\prime}$ is estimates of the effective stress at the onset of unloading, $\mathrm{MPa}, U$ is the unloading parameter, reflect how plastic the sediment is; $A$ and $B$ are parameters calibrated with offset velocity-vs-effective stress data, dimensionless; $V_{p}$ is the compressional velocity, $\mathrm{m} / \mathrm{s}$.

The calculation of horizontal stress is mainly based on the combined spring model, which has a good application effect in the Changning shale gas field. The tectonic stress coefficient $\varepsilon_{h}$ and $\varepsilon_{H}$ are the most important parameter in the model, it can be back-calculation according to the instantaneous shut-in pressure, closure pressure, and acoustic emission test data, based on the back-calculation results, the maximum and minimum horizontal stress are calculated by Young's modulus, Poisson's ratio, pore pressure, and other parameters. The values of $\varepsilon_{h}$ and $\varepsilon_{H}$ in this study are $1 \times 10^{-4}$ and $9 \times 10^{-4}$, respectively. The combined spring model is as follows:

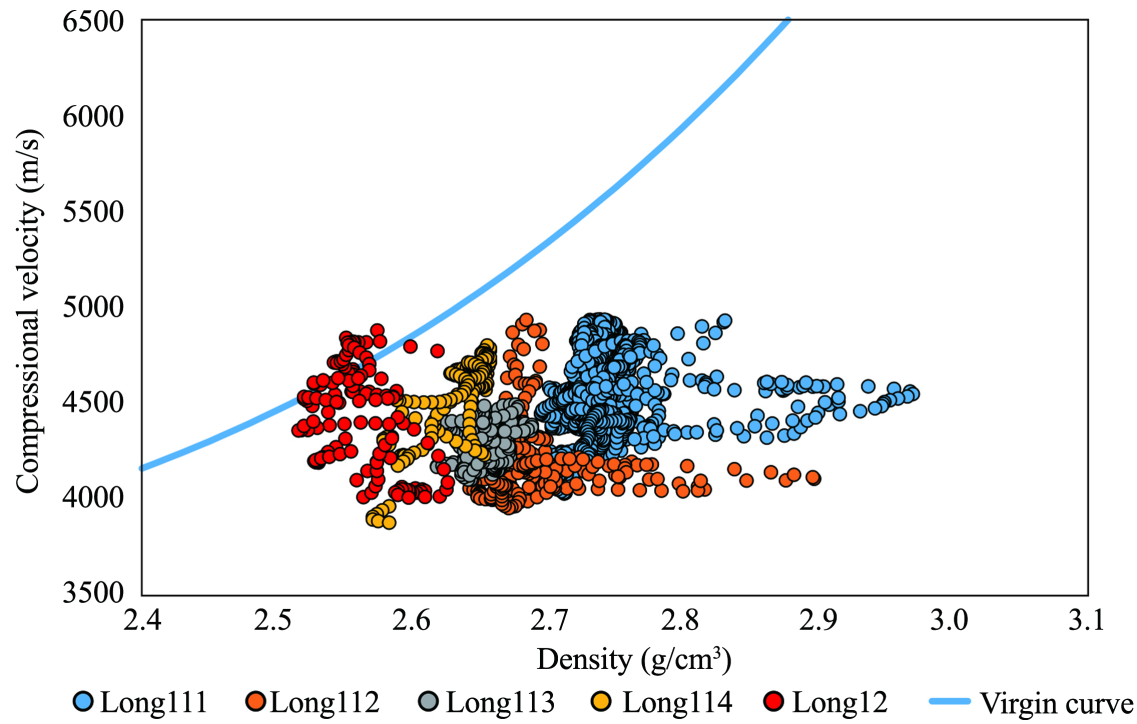

Figure 3. Correlations between compressional velocity and density. 


$$
\begin{aligned}
& S_{h \min }=\frac{v}{1-v} S_{V}+\frac{1-2 v}{1-v} \alpha P_{p}+\frac{E}{1-v^{2}} \varepsilon_{h}+\frac{v E}{1-v^{2}} \varepsilon_{H} \\
& S_{h \max }=\frac{v}{1-v} S_{V}+\frac{1-2 v}{1-v} \alpha P_{p}+\frac{E}{1-v^{2}} \varepsilon_{H}+\frac{v E}{1-v^{2}} \varepsilon_{h}
\end{aligned}
$$

where $S_{h \min }$ is the minimum horizontal stress, MPa; $S_{h \max }$ is the maximum horizontal stress, $\mathrm{MPa} ; v$ is the Poisson's ratio, dimensionless; $E$ is the Young's modulus, $\mathrm{GPa} ; \varepsilon_{h}$ and $\varepsilon_{H}$ are the tectonic stress coefficient, dimensionless; $\alpha$ is the Biot elastic coefficient, which is normally assumed as 1 .

\subsubsection{Collapse Pressure}

The calculation of the collapse pressure is related to the stress states, and rock mechanics parameters. According to the calculation results of in-situ stress, the Luzhou block is in a strike-slip fault stress state, that is, the vertical stress is the intermediate principal stress. The calculation formula of collapse pressure is as follows:

$$
P_{c}=\frac{\eta\left(3 \sigma_{H}-\sigma_{h}\right)-2 S_{0} K+\alpha P_{p}(K-1)}{0.00981 H\left(K^{2}+\eta\right)}
$$

where $P_{c}$ is the collapse pressure, $\mathrm{MPa} ; \eta$ is the nonlinear correction factor of stress, dimensionless; $H$ is the depth, $\mathrm{m} ; S_{0}$ is the rock cohesion, $\mathrm{MPa} ; \alpha$ is the Biot elastic coefficient, which is normally assumed as $1 ; \sigma_{h}$ and $\sigma_{H}$ are the minimum and maximum horizontal stress, $\mathrm{MPa} ; K=\operatorname{ctg}\left(\frac{\pi}{4}-\frac{\varphi}{2}\right)$.

\subsubsection{Three-Dimensional Modeling}

Based on the results of a single well, with seismic inversion attributes as the plane trend constraint, through sequential Gaussian simulation or cokriging simulation method, obtain the distribution characteristics of each parameter in different layers [19] [20] [21] [22].

\section{Results}

\subsection{Organic Matter Abundance and Maturity}

The total organic carbon (TOC) is an important indicator of the organic mat-ter abundance of source rock. The TOC value of high-quality shale is usually greater than $2 \%$. The average TOC of the Long11 sub-member is $3.1 \%$. The TOC of shale from Long 114 to Long111 layer are ranging from $0.25 \%$ to $3.86 \%$ (averaging $2.28 \%$ ), from $1.59 \%$ to $3.04 \%$ (averaging $2.27 \%$ ), from $0.25 \%$ to $5.59 \%$ (averaging $3.22 \%$ ), and from $0.36 \%$ to $9.34 \%$ (averaging $4.29 \%$ ), respectively. Figure 4 showed the characteristic of increasing TOC as the well depth increased, and the TOC values of Long111 and Long112 layers were relatively higher. The vitrinite reflectivity of each layer in the Long 11 sub-member is greater than $2.1 \%$, indicate that the thermal evolution of organic matter during the high-post mature stage. 

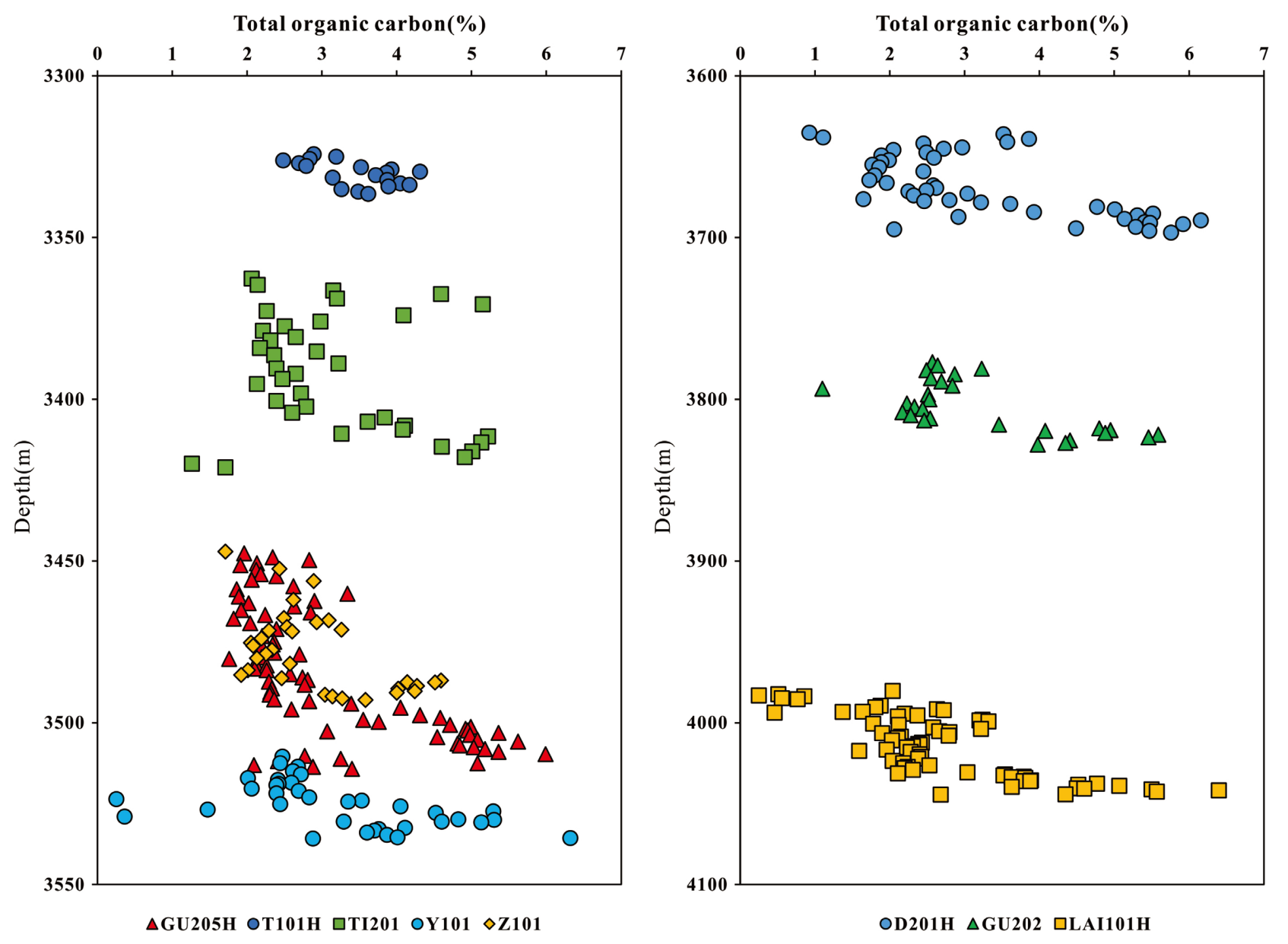

Figure 4. Correlations between depth and total organic carbon.

\subsection{Porosity, Gas Saturation, and Pore Pressure}

Shale pores are the gas storage space in shale gas reservoirs, which have a greater impact on the free gas content, and are a rating index indicating the gas content of the reservoir [23]. Figure 5 shows the depth slice of porosity. The average porosity of the Long11 sub-member is $4.23 \%$. The porosity of shale from Long114 to Long111 layer are ranging from $0.92 \%$ to $6.1 \%$ (averaging $4.1 \%$ ), from $2.85 \%$ to $5.24 \%$ (averaging $4.08 \%$ ), from $1.45 \%$ to $6.79 \%$ (averaging $4.34 \%$ ), and from $0.54 \%$ to $6.94 \%$ (averaging $4.51 \%$ ), respectively.

Gas saturation is one of the main controlling factors of free gas content, the average gas saturation of the Long 11 sub-member is $53.55 \%$. The gas saturation of shale from Long114 to Long111 layer are ranging from $10.58 \%$ to $83.69 \%$ (averaging $43.77 \%$ ), from $20.18 \%$ to $67.42 \%$ (averaging $41.86 \%$ ), from $24.66 \%$ to $89.99 \%$ (averaging $56.25 \%$ ), and from $19.05 \%$ to $89.88 \%$ (averaging $67.47 \%$ ), respectively.

The coefficient of pore pressure is one of the main factors for the high production of shale gas. With the increase of pore pressure, the content of free gas and adsorbed gas in shale will increase. Through the comparison of the test production of appraisal wells and the measured coefficient of pore pressure, it is 


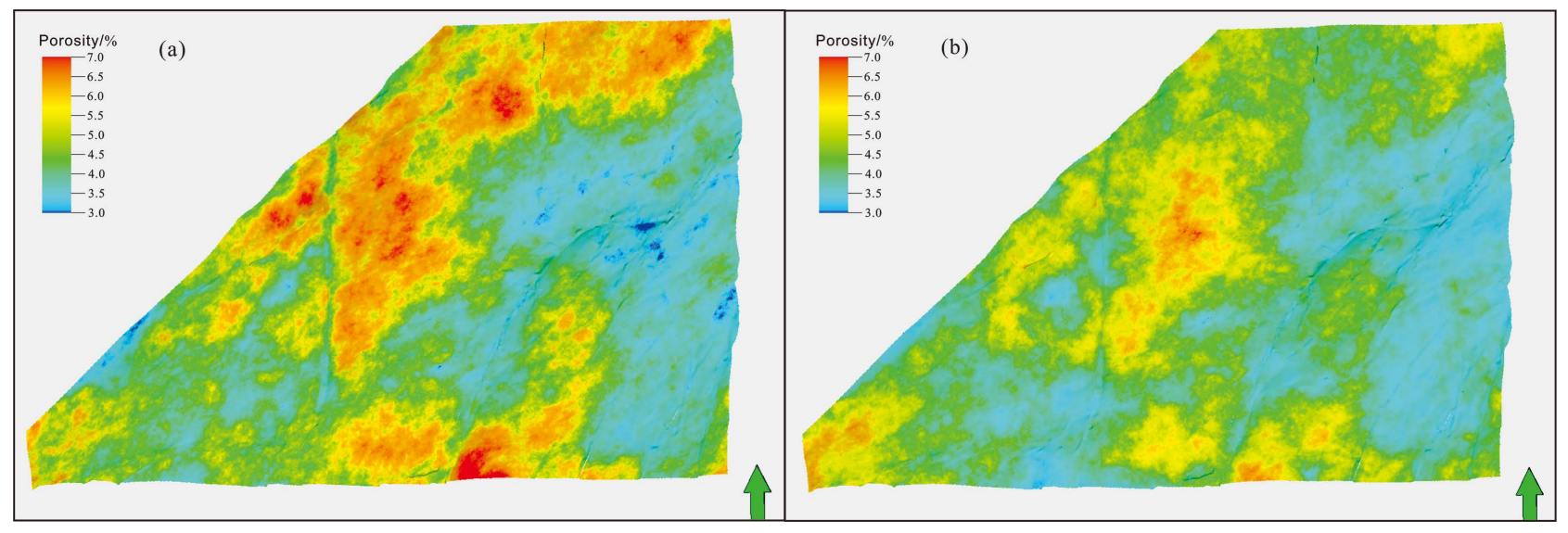

Figure 5. Depth slice of porosity. (a) Long111 formation; (b) Long112 formation.

found that the initial production increases with the increase of the coefficient of pore pressure. The coefficient of pore pressure of the Long11 sub-member is mainly concentrated between $1.94-1.98$.

\subsection{Rock Drillability and ROP}

The drillability level of each layer of the Long11 sub-member is about $4-6$, and the abrasiveness is $2-4$, belonging to the medium-soft stratum, as shown in Figure 6. According to the drill data of 7 horizontal wells that have been drilled in the Luzhou block, when drilling in different layers of the Long11 sub-member with the same drilling parameters and drilling assembly, the ROP (rate of penetrating) is quite different. The ROP of the Long114 and Long113 layers can reach $7-8 \mathrm{~m} / \mathrm{h}$, The Long 112 layer presents the characteristics of low gamma and high drilling time, The ROP is about $5.3 \mathrm{~m} / \mathrm{h}$, on the contrary, the Long111 layer shows the characteristics of high gamma and low drilling time, The ROP is about $6.5 \mathrm{~m} / \mathrm{h}$, as shown in Figure 7.

\subsection{Wellbore Stability}

Combining the collapse pressure, the compressive strength of the stratum, and the actual hole enlargement, to identify the wellbore stability of each layer of the Long11 sub-member. Take Y101H7 well as an example, the collapse pressure of Long114 is relatively high, mainly greater than $1.8 \mathrm{~g} / \mathrm{cm}^{3}$, the corresponding compressive strength is relatively low, mainly less than $180 \mathrm{MPa}$, and the hole enlargement rate is basically greater than $40 \%$, as shown in Figure 8 . The collapse pressure of Long114 is relatively high, mainly greater than $1.8 \mathrm{~g} / \mathrm{cm}^{3}$, the compressive strength is relatively low, mainly less than $180 \mathrm{MPa}$, and the hole enlargement rate is basically greater than $40 \%$. The collapse pressure of Long 111 is relatively low, Average $1.74 \mathrm{~g} / \mathrm{cm}^{3}$, the average compressive strength of the Long111 layer is $193 \mathrm{MPa}$, and the hole enlargement rate is basically within $10 \%$.

\subsection{Reservoir Elastic Parameters}

Young's modulus and Poisson's ratio are used to calculate and characterize the 


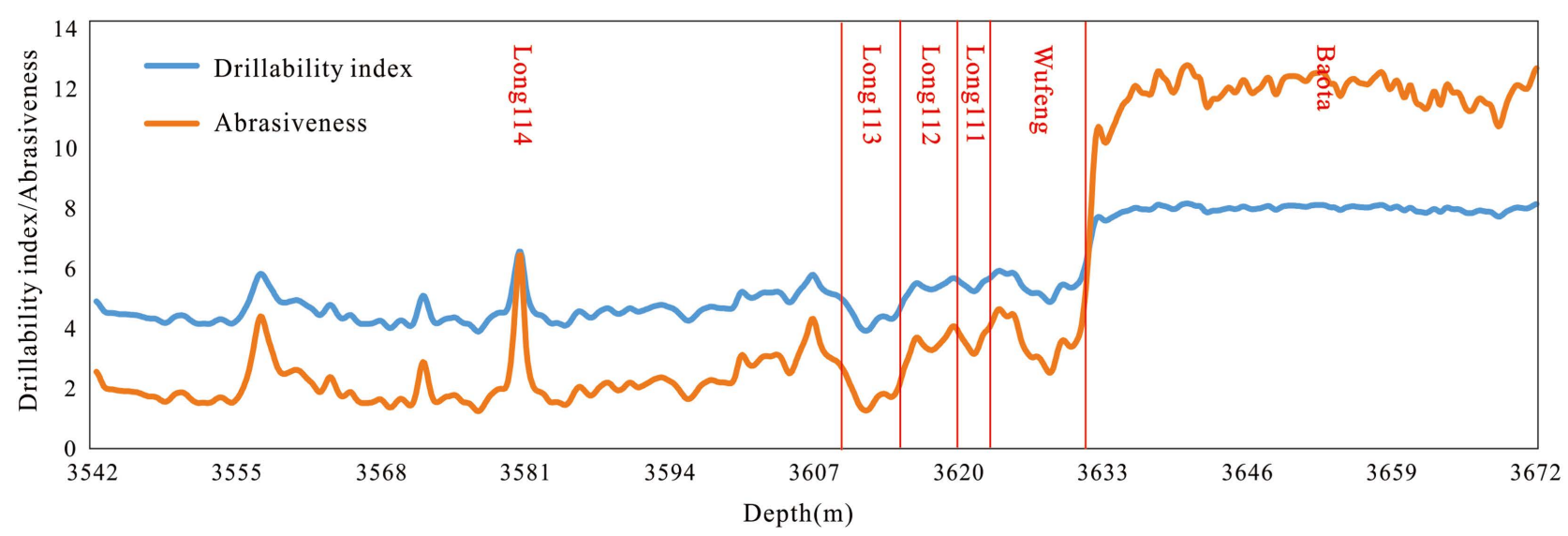

Figure 6. Correlations between depth and drillability index/abrasiveness.

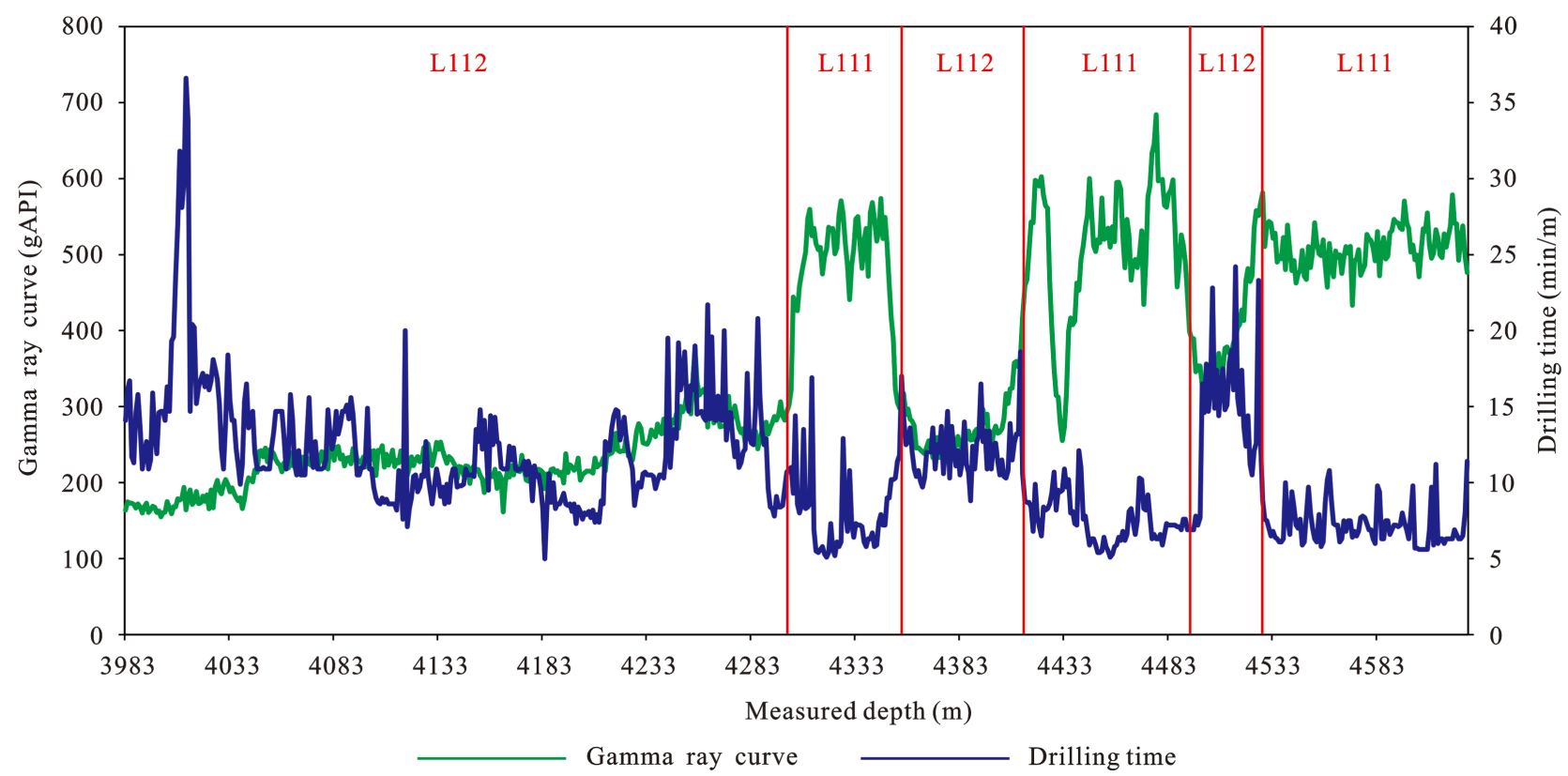

Figure 7. Correlations between measured depth and gamma ray curve.

brittleness of shale. The calculation formula of Young's modulus and Poisson's ratio are as follows:

$$
E_{d y n}=\frac{\rho V_{s}^{2}\left(V_{P}^{2}-4 V_{s}^{2}\right)}{V_{P}^{2}-V_{s}^{2}}
$$

where $E_{d y n}$ is the dynamic elasticity modulus of rock, GPa; $V_{P}$ is the longitudinal wave velocity, $\mu \mathrm{s} / \mathrm{ft}$; $V_{s}$ the shear wave velocity, $\mu \mathrm{s} / \mathrm{ft} ; \rho$ is the rock density, $\mathrm{g} / \mathrm{cm}^{3}$.

$$
V_{d y n}=\frac{V_{P}^{2}-2 V_{s}^{2}}{2\left(V_{P}^{2}-V_{s}^{2}\right)}
$$

where $V_{d y n}$ is the dynamic Poisson's ratio of rock, dimensionless; $V_{P}$ is the longitudinal wave velocity, $\mu \mathrm{s} / \mathrm{ft}$; $V_{s}$ the shear wave velocity, $\mu \mathrm{s} / \mathrm{ft}$.

High-quality shale has obvious characteristics of high Young's modulus and lower Poisson's ratio [5]. Figure 9 shows the depth slice of Young's modulus. 


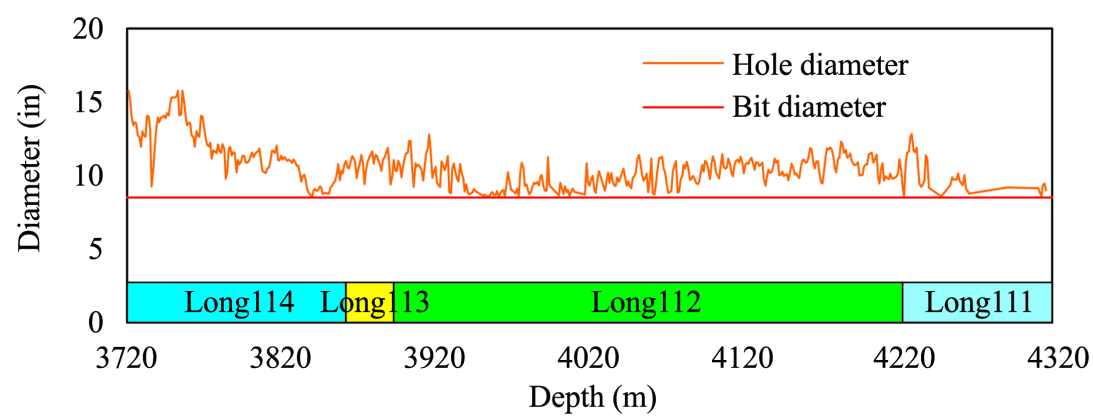

(a)

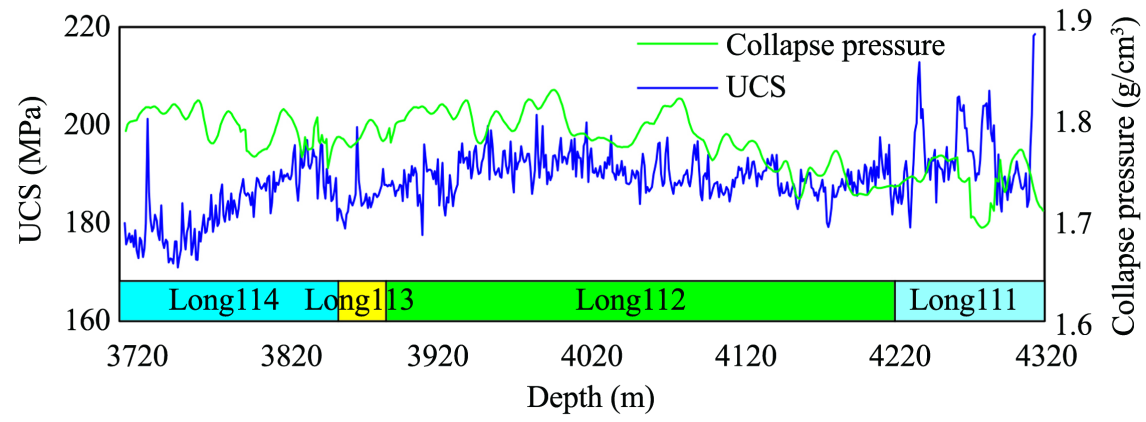

(b)

Figure 8. Wellbore stability parameter of different layers. (a) Correlations between depth and diameter; (b) Correlations between depth and UCS/collapse pressure.

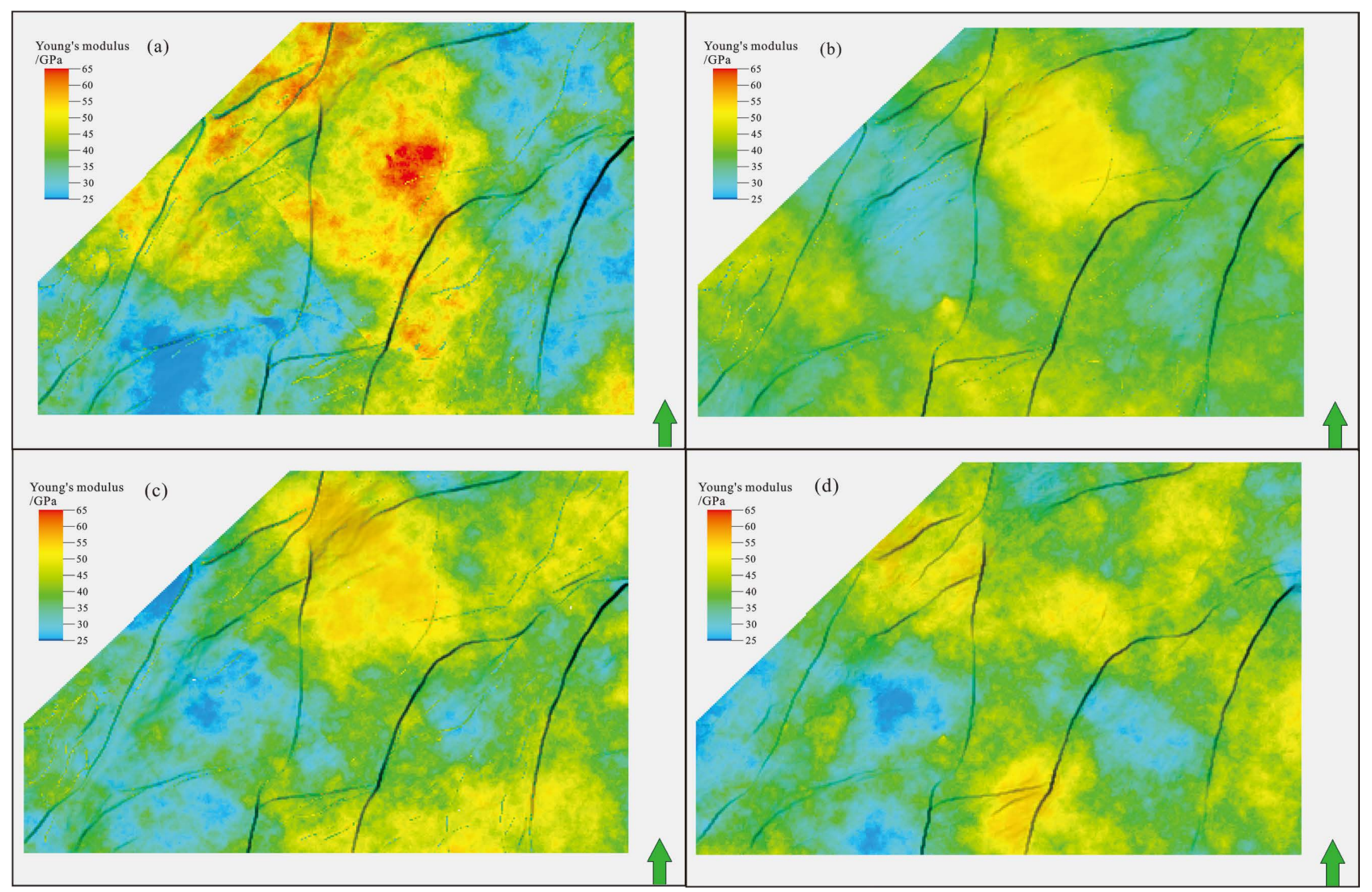

Figure 9. Depth slice of Young's modulus. (a) Long111 formation; (b) Long112 formation; (c) Long113 formation; (d) Long114 formation. 
The average Young's modulus of the Long11 sub-member is $38.87 \mathrm{GPa}$. The Young's modulus of shale from Long114 to Long111 layer are ranging from 25.4 $\mathrm{GPa}$ to $44.69 \mathrm{GPa}$ (averaging $35.24 \mathrm{GPa}$ ), from $30.47 \mathrm{GPa}$ to $40.53 \mathrm{GPa}$ (averaging $35.23 \mathrm{GPa}$ ), from $31.02 \mathrm{GPa}$ to $47.84 \mathrm{GPa}$ (averaging $40.05 \mathrm{GPa}$ ), and from 26.45 GPa to $68.16 \mathrm{GPa}$ (averaging 47.07 GPa), respectively.

The average Poisson's ratio of the Long11 sub-member is 0.17 and the differences between each layer are relatively small. The Poisson's ratio of shale from Long114 to Long111 layer are ranging from 0.11 to 0.27 (averaging 0.17 ), from 0.12 to 0.24 (averaging 0.17 ), from 0.13 to 0.22 (averaging 0.17 ), and from 0.12 to 0.26 (averaging 0.18 ), respectively.

\subsection{Mineral Composition and Brittleness Index}

Figure 10 shows the X-ray diffraction analysis result, it indicated that the shale mineral composition of the Long11 sub-member in the Luzhou block are mainly clay minerals, quartz, dolomite, plagioclase, and calcite. The clay mineral content is the highest, with an average of $39.04 \%$. And the content of quartz, calcite, and dolomite are $36.35 \%, 4.52 \%$, and $7.47 \%$, respectively. There is a certain difference in the mineral composition of Long114 to Long111 sublayer, the content of clay minerals gradually decreases, and the content of quartz gradually increases from shallow to deep.

The brittle mineral contents in the shale reservoir control the fracturing ability of the shale, and the brittleness index can be calculated by the following formula:

$$
I B=\frac{Q t z}{Q t z+C a l+c l a y} \times 100 \%
$$

The average brittleness index of the Long11 sub-member is $45.49 \%$, and the average brittleness index of shale from Long 114 to Long 111 layer are $31.53 \%$, $40.08 \%, 50.52 \%$, and $62.53 \%$, respectively.

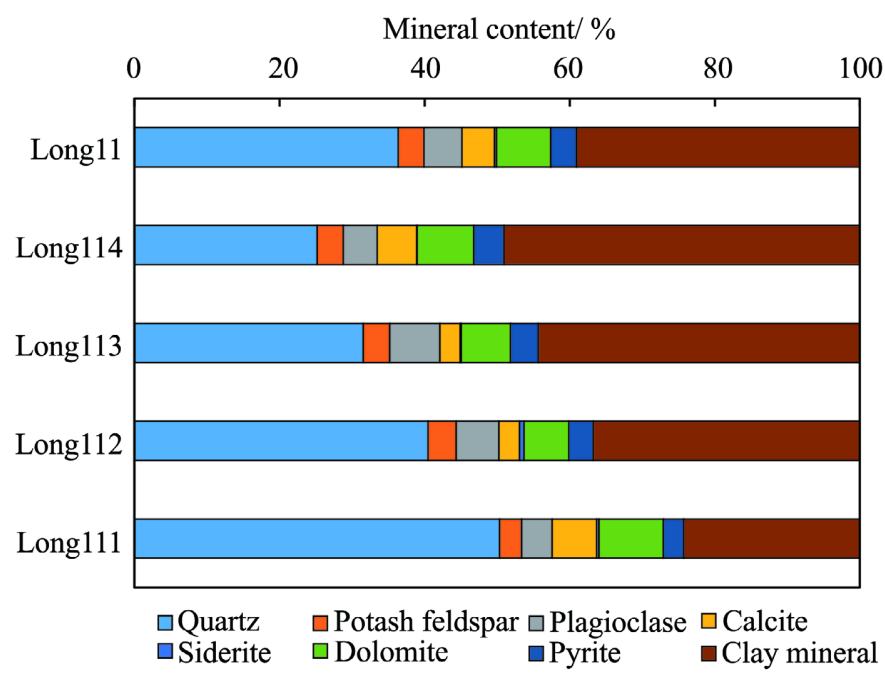

Figure 10. Mineral composition of different layers. 


\subsection{Horizontal Stress and Fracture}

The stress difference coefficient determines the fracture geometry after fracturing. The smaller the stress difference coefficient $(0-0.3)$, the easier it is to form a complex fracture network after hydraulic fracturing. Figure 11 shows the stress difference coefficient of shale from Long114 to Long111 layer are ranging from 0.04 to 0.16 (averaging 0.10 ), from 0.06 to 0.14 (averaging 0.11 ), from 0.04 to 0.16 (averaging 0.13 ), and from 0.06 to 0.15 (averaging 0.12 ), respectively. The horizontal stress difference of shale from Long114 to Long111 layer are ranging from $5 \mathrm{MPa}$ to $16 \mathrm{MPa}$ (averaging $8.85 \mathrm{MPa}$ ), from $6 \mathrm{MPa}$ to $15 \mathrm{MPa}$ (averaging 9.07 $\mathrm{MPa}$ ), from $7 \mathrm{MPa}$ to $17 \mathrm{MPa}$ (averaging 9.27 $\mathrm{MPa}$ ), and from $7 \mathrm{MPa}$ to 15 $\mathrm{MPa}$ (averaging 9.17 $\mathrm{MPa}$ ), respectively.

The developing degree of fractures is an important factor in determining whether hydraulic fracturing can form a large-scale fracture network. Meanwhile, the development of natural fractures makes it easy for free gas to accumulate to achieve a high-yield well. Ac-cording to core fracture description, the high angle fracture density of Long113 to Long111 layer is generally between 3 10 per $0.5 \mathrm{~m}$. Discrete fracture network modeling combined with identified imaging fracture is adopted to build natural fracture model as shown in Figure 12, The results showed that natural fractures in the lower reservoirs (Long113 to

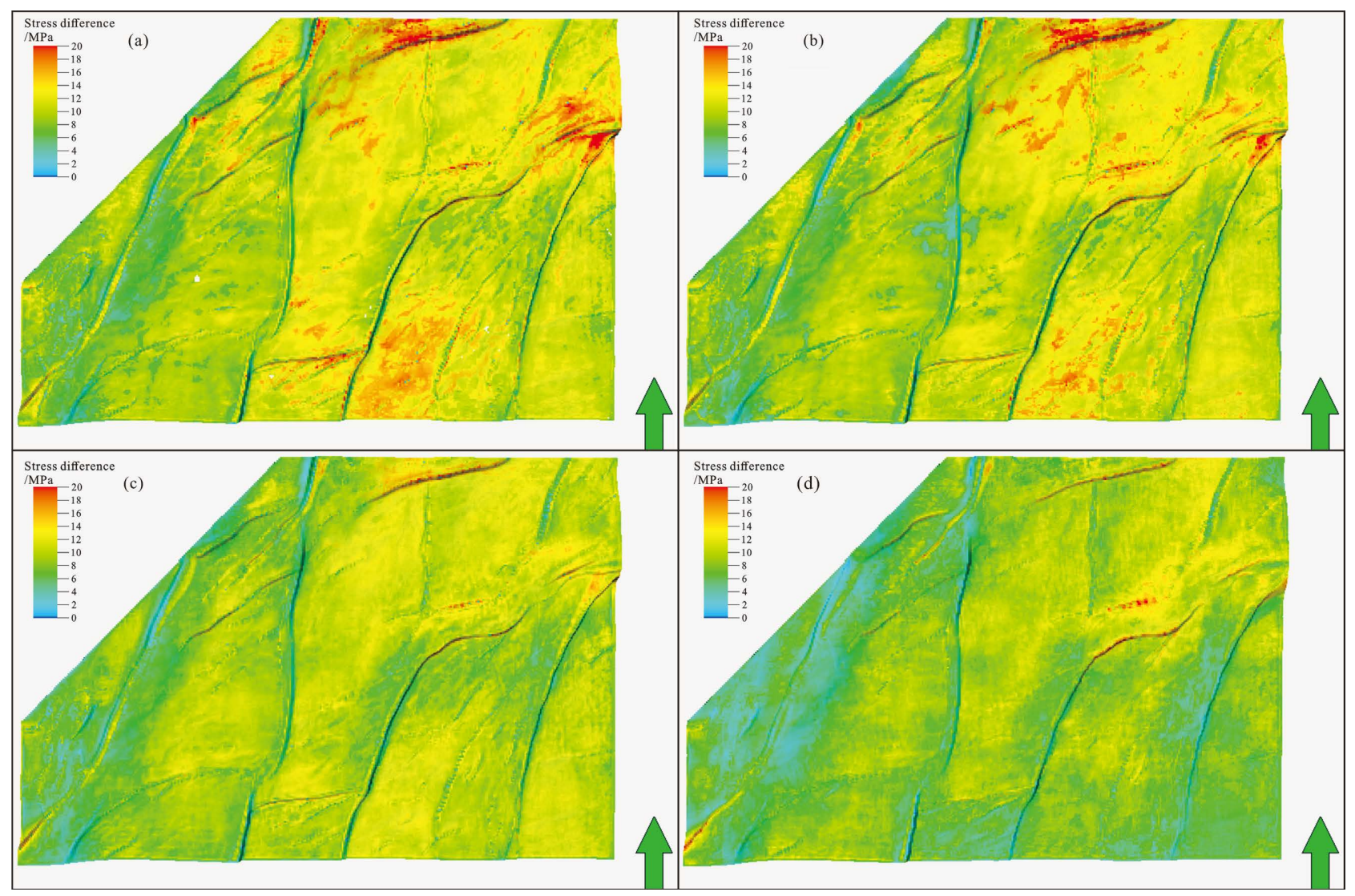

Figure 11. Depth slice of stress difference (a) Long111 formation; (b) Long112 formation; (c) Long113 formation; (d) Long114 formation. 


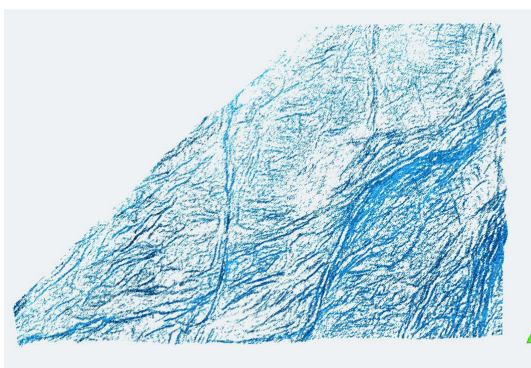

(a)

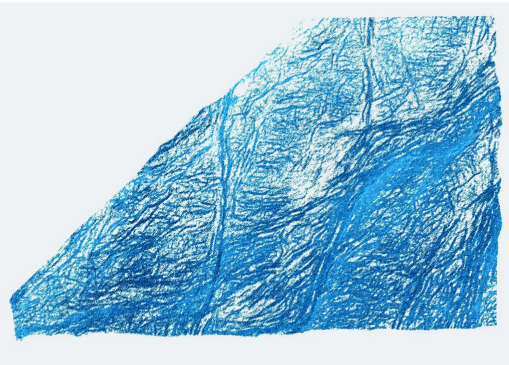

(b)

Figure 12. Natural fracture model. (a) Long114 layer; (b) Long113 to Long111 layer.

Long111 layer) are more developed, the length of natural fractures is mainly distributed in $50-80 \mathrm{~m}$, the inclination of natural fractures is mainly greater than 50 degrees, and the number of high-angle fractures is obviously more than that of low-angle fractures.

\section{Discussion}

\subsection{Geological Sweet Spots}

According to the previous research and Chinese energy industry standards NB/T 10398-2020, parameters on shale gas geological sweet spots evaluation mainly include total organic carbon content, thermal maturity, total porosity, gas saturation, permeability, pore pressure, and reservoir thickness, etc. Through the study of the correlation between gas production and sweet spot parameters, it is found that the total organic carbon content, porosity, gas saturation, and pore pressure, have a greater impact on gas production.

It is generally accepted that the samples with TOC ranging $>3.0 \%, 3.0 \%-2.0 \%$, and $<2.0$ are regarded to be good, medium, and poor reservoirs, respectively. The experimental results show that the average TOC of samples from Long111 and Long112 layers are greater than 3.0\%, indicating they are good reservoirs. Whereas the average TOC of samples from Long113 and Long114 layers are $2.27 \%$ and $2.28 \%$, respectively, indicating they are medium reservoirs, as shown in Table 2 .

Good, medium and poor reservoirs are associated with porosity of $>4.0 \%$, $4.0 \%-2.0 \%,<2.0$, respectively. The average porosity of samples from Long 114 to Long111 layers are greater than $4.0 \%$, and the Long 111 layer is up to $4.51 \%$, indicating they are good reservoirs.

Samples with gas saturation $\geq 60 \%$ are considered as good reservoirs, whereas with gas saturation ranging $60 \%-50 \%$, and $<50 \%$ are regarded to be medium, and poor reservoirs, respectively. The experimental results show that the average gas saturation of samples from Long 111 layer is greater than $60 \%$, indicating it is good reservoir. whereas the average gas saturation of samples from Long 113 and Long114 layers are $41.86 \%$ and $43.77 \%$, respectively, indicating they are poor reservoirs. The coefficient of pore pressure of the Long114 to Long111 layers has little difference., and the abnormal high pressure is conducive to form the high-yield gas reservoirs. 
The Long112 and Long111 layers are far superior to the Long113 and Long114 layers in terms of TOC, porosity, and gas saturation, and the Long111 layer is the best reservoir. Ensure the drilled rate of the Long111 layer under the fixed horizontal section length is an effective way of increasing production.

\subsection{Drilling Sweet Spots}

Drilling sweet spots were often overlooked in previous evaluations of shale gas sweet spots, including the reservoir, geomechanics, and engineering parameters that have an important impact on drilling safety and drilling efficiency.

Wellbore instability in shale reservoirs poses a serious threat to drilling safety, which can cause sticking and even burying drill tools. Experimental and modeling results show that higher collapse pressure and lower compressive strength correspond to larger hole enlargement rate, which means a greater risk of wellbore instability. Long111 layer has lower collapse pressure, higher compressive strength, smaller hole enlargement rate, and relatively low risk of wellbore instability. Drilling in the Long111 layer can effectively re-duce the risk of wellbore instability.

The ROP is the most intuitive indicator of drilling efficiency. The ROP of Long114 and Long113 layers are relatively high, and the Long112 layer is the lowest. Calculated based on 1500-meter horizontal sections, drilling in the Long111 and Long114 layers can save more than 50 and 80 hours of net drilling time compared to drilling in the Long112 layer, respectively.

Considering drilling safety and drilling efficiency comprehensively, drilling in the Long111 layer is conducive to reducing the risk of drilling operation and ensuring drilling efficiency to a certain extent.

\subsection{Fracturing Sweet Spots}

The fracturing sweet spots mainly evaluate whether the shale reservoir is easy to fracturing transformation or the effect of fracturing transformation. The parameters mainly include brittleness index, stress difference coefficient, and developing degree of fractures.

Table 2. Shale gas geological sweet spots parameters of different layers.

\begin{tabular}{|c|c|c|c|}
\hline Formation & TOC/\% & Porosity/\% & Gas saturation/\% \\
\hline \multirow{2}{*}{ Long114 } & $\underline{0.25-3.86}$ & $\underline{0.92-6.1}$ & $\underline{10.58-83.69}$ \\
\hline & $2.28(92)$ & $4.1(92)$ & $43.77(92)$ \\
\hline \multirow{2}{*}{ Long113 } & $\underline{1.59-3.04}$ & $\underline{2.85-5.24}$ & $\underline{20.185-67.42}$ \\
\hline & $2.27(48)$ & $4.08(48)$ & $41.86(48)$ \\
\hline \multirow{2}{*}{ Long112 } & $\underline{0.25-5.59}$ & $\underline{1.45-6.79}$ & $24.66-89.99$ \\
\hline & $3.22(84)$ & $4.34(84)$ & $56.25(84)$ \\
\hline \multirow{2}{*}{ Long111 } & $\underline{0.36-9.34}$ & $\underline{0.54-6.94}$ & $\underline{19.05-89.88}$ \\
\hline & $4.29(82)$ & $4.51(92)$ & $67.47(92)$ \\
\hline
\end{tabular}

Annotation: The data format in the table is: (maximum value - minimum value)/mean value (sample numbers). 
It is generally accepted that the samples with Young's modulus ranging $>30$ $\mathrm{GPa}, 30-20 \mathrm{GPa}$, and $<20 \mathrm{GPa}$ are regarded to be good, medium, and poor reservoirs, respectively. The experimental results show that the average Young's modulus of samples from Long114 to Long111 layers are greater than $30 \mathrm{GPa}$, and the Long111 layer is up to $47.07 \mathrm{GPa}$, indicating they are good reservoirs, as shown in Table 3.

Good, medium and poor reservoirs are associated with Poisson's ratio of $<0.2$, $0.2-0.25,>0.25$, respectively. The average porosity of samples from Long114 to Long 111 layers are greater than $4.0 \%$, and the Long 111 layer is up to $4.51 \%$, indicating they are good reservoirs. The differences of the average Poisson's ratio of each layer are relatively small, all layers belong to good reservoirs.

Samples with brittleness index $\geq 60 \%$ are considered as good reservoirs, whereas with gas saturation ranging $60 \%-40 \%$, and $<40 \%$ are regarded to be medium, and poor reservoirs, respectively. The experimental results show that the average brittleness index of samples from Long111 layer is greater than $60 \%$, indicating it is good reservoir. where-as the average brittleness index of samples from Long 114 layer is $31.57 \%$, indicating it is poor reservoirs.

It is generally accepted that the samples with stress difference coefficient ranging $0-0.3,0.3-0.5$, and $>0.5$ are regarded to be good, medium, and poor reservoirs, respectively. The average stress difference coefficient of samples from Long114 to Long111 layers are less than 0.13 , indicating they are good reservoirs.

The natural fractures in the lower reservoirs (Long113 to Long111 layer) are more developed, the high angle fracture density is generally between 3 - 10 per $0.5 \mathrm{~m}$, and the length of natural fractures is mainly distributed in $50-80 \mathrm{~m}$. The characteristics are conducive to form a complex fracture network.

Long111 layer is the best fracturing sweet spot which has a high Young's modulus and brittleness index, and has developed natural fractures. Integrate the Long111 layer as much as possible when segmenting, to make it fully fracturing transformation. The perforation position should be selected in the low value area of the minimum horizontal stress, and the stress difference coefficient between

Table 3. Shale gas fracturing sweet spots parameters of different layers.

\begin{tabular}{|c|c|c|c|c|}
\hline Formation & $\begin{array}{c}\text { Young's } \\
\text { modulus/Gpa }\end{array}$ & $\begin{array}{l}\text { Poisson's } \\
\text { ratio }\end{array}$ & $\begin{array}{l}\text { Brittleness } \\
\text { index/\% }\end{array}$ & $\begin{array}{l}\text { Stress difference } \\
\text { coefficient }\end{array}$ \\
\hline Long114 & $\frac{25.4-44.69}{3524(55)}$ & $\frac{0.11-0.27}{0.17(55)}$ & $\frac{25.4-36.47}{3157(21)}$ & $\frac{0.04-0.16}{0.04(24)}$ \\
\hline Long113 & $\frac{30.47-40.53}{35.23(46)}$ & $\frac{0.13-0.22}{0.17(46)}$ & $\frac{27.7-46.5}{40.08(23)}$ & $\frac{0.06-0.14}{0.11(22)}$ \\
\hline Long112 & $\frac{31.02-47.84}{40.05(60)}$ & $\frac{0.12-0.24}{0.17(60)}$ & $\frac{38.4-63.87}{50.52(28)}$ & $\frac{0.04-0.16}{0.13(28)}$ \\
\hline Long111 & $\frac{26.45-68.16}{47.07(62)}$ & $\frac{0.12-0.26}{0.18(62)}$ & $\frac{54.16-77.21}{62.35(30)}$ & $\frac{0.06-0.15}{0.12(30)}$ \\
\hline
\end{tabular}

Annotation: The data format in the table is: (maximum value - minimum value)/mean value (sample numbers). 
perforation clusters should be as small as possible. The large-scale fracturing section should be designed in the sweet spot when optimizing the scale of fracturing.

\section{Conclusions}

In this study, the geological and engineering sweet spots of the shale gas reservoir in the Luzhou block are characterized by core experiment data and 3D geomechanical model. The main conclusions in this work can be drawn as follows.

1) The Long111 layer is the best reservoir of geological sweet spot which has high TOC, porosity, and gas saturation. Ensuring the drilled rate of the Long111 layer under the fixed horizontal section length is an effective way of increasing production.

2) Considering drilling safety and drilling efficiency comprehensively, drilling in the Long111 layer is conducive to reducing the risk of drilling operation and ensuring the drilling efficiency to a certain extent.

3) Long111 layer is the best fracturing sweet spot which has a high Young's modulus and brittleness index, and has developed natural fractures. The large-scale fracturing section should be designed in the sweet spot when optimizing the scale of fracturing.

4) The Long111 layer is the optimal reservoir section of the Longmaxi Formation. Ensure the drilled rate of the Long111 layer and maximize the length of the horizontal section can obtain higher production.

\section{Acknowledgements}

This paper is supported by the Major Engineering Technology Field Test Project of China National Petroleum Corporation (Nos. 2019F-31).

\section{Conflicts of Interest}

The authors declare no conflicts of interest regarding the publication of this paper.

\section{References}

[1] Xie, J., Zhang, H.M., She, C.Y., Li, Q.R., Fan, Y. and Yang, Y. (2017) Practice of Geology-Engineering Integration in Changning State Shale Gas Demonstration Area. China Petroleum Exploration, 22, 21-28.

[2] Xie, J. (2018) Practices and Achievements of the Changning-Weiyuan Shale Gas National Demonstration Project Construction. Natural Gas Industry, 38, 1-7.

[3] Ma, X.H. (2017) A Golden Era for Natural Gas Development in the Sichuan Basin. Natural Gas Industry, 47, 1-10.

[4] Zou, C.N., Dong, D.Z. and Wang, Y.M. (2015) Shale Gas in China: Characteristics, Challenges and Prospects (I). Petroleum Exploration and Development, 42, 689-701. https://doi.org/10.1016/S1876-3804(15)30072-0

[5] Ma, X.H., Xie, J. and Yong, R. (2020) Geological Characteristics and High Produc- 
tion Control Factors of Shale Gas in Silurian Longmaxi Formation, Southern Sichuan Basin, SW China. Petroleum Exploration and Development, 47, 1-15. https://doi.org/10.1016/S1876-3804(20)60105-7

[6] Yang, H.Z., Zhao, S.X. and Liu, Y. (2019) Main Controlling Factors of Enrichment and High-Yield of Deep Shale Gas in the Luzhou Block, Southern Sichuan Basin. Natural Gas Industry, 39, 55-63.

[7] Buller, D., Hughes, S.N. and Market, J. (2010) Petrophysical Evaluation for Enhancing Hydraulic Stimulation in Horizontal Shale Gas Wells. The SPE Annual Technical Conference and Exhibition, Florence, 20-22 September 2010, SPE-132990-MS. https://doi.org/10.2118/132990-MS

[8] Pan, R.F., Gong, Q. and Yan, J. (2016) Elements and Gas Enrichment Laws of Sweet Spots in Shale Gas Reservoir: A Case Study of the Longmaxi Formation in Changning Block, Sichuan Basin. Natural Gas Industry, 36, 7-13. https://doi.org/10.1016/j.ngib.2016.05.003

[9] Ross, D.J.K. and Bustin, R.M. (2007) Shale Gas Potential of the Lower Jurassic Gordondale Member, Northeastern British Columbia, Canada. Bulletin of Canadian Petroleum Geology, 55, 51-75. https://doi.org/10.2113/gscpgbull.55.1.51

[10] Guo, X.Y., Chen, Y.C., Zhang, J., Man, L., Zheng, H.Q. and Tong, X.J. (2015) Assessment Index Selection and Weight Determination of Shale Gas Plays: A Case Study of Marine Shale in the Sichuan Basin. Natural Gas Industry, 35, 57-64.

[11] Zhao, S.X., Yang, Y.M. and Zhang, J. (2016) Micro-Layers Division and Fine Reservoirs Contrast of Lower Silurian Longmaxi Formation Shale, Sichuan Basin, SW China. Nature Gas Geoscience, 27, 78-95.

[12] Zhang, Y., Jiang, S., He, Z., Li, Y., Xiao, D., Chen, G. and Zhao, J. (2021) Coupling between Source Rock and Reservoir of Shale Gas in Wufeng-Longmaxi Formation in Sichuan Basin, South China. Energies, 14, 2679.

https://doi.org/10.3390/en14092679

[13] Zheng, M., Tang, H., Li, H., Zheng, J. and Jing, C. (2020) Geomechanical Analysis for Deep Shale Gas Exploration Wells in the NDNR Blocks, Sichuan Basin, Southwest China. Energies, 13, 1117. https://doi.org/10.3390/en13051117

[14] Zeng, Y., Chen, Z. and Bian, X. (2016) Breakthrough in Staged Fracturing Technology for Deep Shale Gas Reservoirs in SE Sichuan Basin and Its Implications. Natural Gas Industry, 3, 45-51. https://doi.org/10.1016/j.ngib.2016.02.005

[15] Ma, X., Wang, H. and Zhou, S. (2021) Deep Shale Gas in China: Geological Characteristics and Development Strategies. Energy Reports, 7, 1903-1914. https://doi.org/10.1016/j.egyr.2021.03.043

[16] Zheng, S., Xie, X. and Luo, L. (2029) Fast and Efficient Drilling Technologies for Deep Shale Gas Horizontal Wells in the Sichuan Basin: A Case Study of Well Lu 203. Natural Gas Industry, 39, 88-93.

[17] Yan, W., Feng, M., Wang, Y., Liu, S., Li, K., Zhao, X. and Liang, W. (2018) The Application of a New Neutron Induced Gamma Ray Spectroscopy Tool in Evaluation the Shale Gas in Fuling Shale Gas Field. Open Journal of Yangtze Oil and Gas, 3, 93-103. https://doi.org/10.4236/ojogas.2018.32008

[18] Bowers, G.L. (1995) Pore Pressure Estimation from Velocity Data: Accounting for Overpressure Mechanisms besides Under-Compaction. SPE Drilling \& Completion, 10, 89-95. https://doi.org/10.2118/27488-PA

[19] Xie, J., Qiu, K.B. and Zhong, B. (2017) Construction of 3D Geomachanical Model for Development of a Shale Gas Reservoir in Sichuan Basin. The SPE Russian Petroleum Technology Conference, Moscow, 16-18 October 2017. 
https://doi.org/10.2118/187828-RU

[20] Qiu, K., Marsden, R. and Solovyov, Y. (2015) Downscaling Geomechanics Data for Thin Beds Using Petrophysical Techniques. The SPE Middle East Oil \& Gas Show and Conference, Kingdom of Bahrain, 12-15 March 2005, SPE 93605.

https://doi.org/10.2118/93605-MS

[21] Qiu, K., Felgueroso, J.G. and Lalinde, G. (2008) Geomechanics Enables the Success of Horizontal Well Drilling in Libya: A Case Study. The IADC/SPE Drilling Conference, Orlando, 4-6 March 2008, IADC/SPE 111384.

https://doi.org/10.2118/111384-MS

[22] Wu, Q., Liang, X. and Xian, C.G. (2015) Geoscience-to-Production Integration Ensures Effective and Efficient South China Marine Shale Gas Development. China Petroleum Exploration, 20, 1-23.

[23] Ambrose, R.J., Hartman, R.C. and Campos, M.D. (2010) New Pore-Scale Considerations for Shale Gas in Place Calculations. SPE Unconventional Gas Conference, Pittsburgh, 23-25 February 2010, SPE-131772-MS.

https://doi.org/10.2118/131772-MS 